\title{
UNIFORM SELECTION ALGORITHM FOR BIG DATA RANDOM PROCESS AND REPEAT USE BASED ON TOPOLOGICAL LOCATION
}

\author{
Jaehoon You ${ }^{1}$ and Cheong Youn ${ }^{2 *}$ \\ ${ }^{1}$ Department of Computer Engineering, Chung-Nam National University, Korea \\ ${ }^{2}$ Department of Computer Engineering, Chung-Nam National University, Korea \\ ${ }^{1}$ heroyou@cnu.ac.kr, ${ }^{2}$ cyoun@cnu.ac.kr
}

Abstract-Since the information revolution, massive online content has been placed on the Internet, and it has become possible to use it indefinitely as long as it is in demand. In the past, such content was disposable, but products having higher frequency of use have been explosively increasing because of the development of technology. Existing algorithms are biased towards simple sorting and aggregation. In this paper, we define an algorithm based on topological location that guarantees uniform selection, weight selection, and fast processing speed for problems that occur from mass consumption and repeatable reuse of tangible and intangible products, and we attempt to demonstrate these features through experiments. Disadvantages of the simple random sampling method are that it cannot guarantee a uniform selection and that it does not offer a way to select using different weights. Hence, the proposed algorithm assigns priority to the uniform probability distribution without making an assumption about the distribution. Further, by narrowing the selection range by digitizing the selection frequency to the topographical position, uniformity of random selection is ensured, and the processing speed for mass selection can be dramatically increased.

Keywords - Algorithm, Random, Big data, Select, Topography, Process

\section{INTRODUCTION}

\subsection{BACKGROUND}

After the third industrial revolution, a large number of products have been massproduced, and today, with the development of technology, even the finer work processes can be replaced by machines. In addition, unmanned automation systems and industrial robots were used to achieve breakthroughs in the mass production system. Mass production is the same as mass consumption and eventually leads to waste of resources.

There are three main types of product consumption. First, there are non-reusable products with expiration dates. For example, if the food items such as vegetables and meats used in restaurants all have different expiration dates, finding and selecting the product with the shortest expiration date is a method of efficiently using the purchased product.

The second type is of products that are reusable but have a limited frequency of use. For example, rechargeable batteries used in everyday life and laser printer toner have a common feature, which is that they can be used repeatedly, but there are limits to the frequency of use. A simple method for managing products that can be used

Received: March 29, 2019

Reviewed: June 4, 2019

Accepted: June 7, 2019 
repeatedly but that have a limited frequency of use is to use them one by one, and when all have been used, to buy new products. However, this method is not effective if there is an expiration date and if several products are used at the same time. For example, if you want to select three batteries from 20 rechargeable batteries, you can choose three batteries at random. When all three batteries are used up, you can select another three and then charge the rechargeable batteries. The ideal combination when repeating this process several times is to make the usage frequency of the 20 rechargeable batteries uniform. A technique for managing this is to simply create a usage record for each rechargeable battery and select the rechargeable battery to be used next. However, if the total number is increased and the number to be selected at the same time is not constant, the complexity required to achieve uniform selection increases. For example, a case for which the quantity is large, the selection quantity is variable, and uniform selection frequency is important is NAND flash, which is used in a solid-state device with a limited number of writes. When writing, it is effective to uniformly use the entire NAND flash as much as possible [1]. However, it is not easy to manage a uniform number of writes for all bits, as even the $128 \mathrm{~GB}$ products have a bit count of $1024 \mathrm{~Gb}$ [2].

The third category is products that can be used indefinitely. In the case of online content such as a service providing test questions, it is necessary to extract several test questions at the same time. In addition, it is necessary to have the test questions with low exposure appear first and to provide the user with a random and uniform frequency of test questions If a few words or test questions appear to be repeating too intensively, the effectiveness of learning can be decreased.

A simple method for uniform selection is to use a uniform random number generator, and when several items are selected at the same time, it should be made to select without duplicates. However, if this method is repeated several times, the overall selection may appear uniform, but the standard deviation of the selection frequencies is not small, and it takes a considerable amount of time to repeat until the frequencies for all the selections become constant Another way to overcome the problem is to use the selection frequency and select the least selected ones first, but problems arise when there are many numbers If the number increases, it will take a long time to arrange them because each time a selection is made, they must be sorted and randomly selected from using the selection frequencies [3], [4].

\subsection{RESEARCH OBJECTIVES}

Our uniform random generator is a method designed to solve various problems that occur in selection and reuse, and it is a method for fast and accurate uniform selection. It topographically analyzes the frequency of selection of the selected location to determine the point and direction to select. This feature does not require alignment because it adjusts the selection position with a simple topographic feature from the first selected location. Just by following simple selection rules, the selection frequencies are automatically kept uniform, resulting in superior speed and stability [5], [6].

Although the proposed method is for uniform selection, it is also possible to choose to have the form of a specific distribution. The method is to apply a weight to the selection frequency to make a selection with a modified selection frequency. For example, to increase the frequency of selection and improve the selection, the actual selection frequency could be multiplied by 0.8 to appear as the selection frequency. Conversely, in order to lower the frequency of selection, the actual selection frequency could be multiplied by 1.5 and the result used as the selection frequency [7], [8].

An example of how this technique can be applied is in the selection of a product with an expiration date when the product has a relatively short expiration. In this 
case, it is possible to make it appear as though the selection frequency is lower than the actual selection frequency, thereby increasing its chance of being selected.

Another example is in writing to a NAND flash, the main accessory of an SSD, which should be as uniform as possible because of the frequency limitations. Because there are so many NAND flashes in an SSD, they must be divided into blocks. However, since the number of blocks is very large, this simple algorithm is suitable for making the write frequency uniform as a whole.

In this paper, we propose a topological location-based selection algorithm that can be used throughout the consumer industry to prevent unnecessary waste of finite resources, reduce the amount of time and energy consumed by mass consumption, and maximize the lifespan of the product by increasing the efficiency of consumption.

\section{METHODS}

\subsection{PRELIMINARY CONCEPTS}

If $\mathrm{m}$ balls are randomly selected each time from a basket of $\mathrm{K}$ balls without any restrictions on the number of times each ball is selected, even with the use of a uniform random number generator uniformity cannot be guaranteed because there is no control; in addition, balls selected previously can subsequently be selected again. When randomness and uniformity are guaranteed, and if the process of randomly selecting $\mathrm{m}$ balls from a basket of $\mathrm{K}$ balls is done $\mathrm{n}$ times, and if the selection frequencies for each ball become similar, then the probability distribution for the frequency of selection becomes a binomial distribution. In this case, the probability (p) of selection is given by the formula [9].

$$
p=\frac{m}{K}
$$

The probability of the ith ball being chosen $\mathrm{x}$ times in $\mathrm{n}$ selections is

$$
p\left(x_{i} \mid n\right)=\left(\begin{array}{c}
n \\
x_{i}
\end{array}\right) p^{x_{i}}(1-p)^{n-x_{i}}
$$

and the expected value and standard deviation of this formula are, respectively,

$$
E\left(x_{i}\right)=n p, \quad \sigma_{x_{i}}=\sqrt{n p(1-p)}
$$

For example, when the process of selecting three balls from 10 balls is done four times, the expected value of the number of times each ball is chosen becomes $4 \times 0.3=1.2$. Table I shows the frequency of selection for each ball. By the fourth iteration, most of the balls were selected once, and only two balls were selected twice. After repeating this experiment $\mathrm{n}$ times, the probability of the frequency of selection is found by multiplying all the probability values since each ball is selected independently:

$$
\prod_{i=1}^{K}\left(\begin{array}{c}
n \\
x_{i}
\end{array}\right) p^{x_{i}}(1-p)^{n-x_{i}}
$$

Table I. Four Iterations of Selecting Three Balls From Ten Balls

\begin{tabular}{|l|l|l|l|l|l|l|l|l|l|l|}
\hline & No.1 & No.2 & No.3 & No.4 & No.5 & No.6 & No.7 & No.8 & No.9 & No.10 \\
\hline 1TIME & 1 & 0 & 1 & 0 & 0 & 0 & 0 & 0 & 1 & 0 \\
\hline 2TIME & 1 & 1 & 1 & 0 & 0 & 0 & 0 & 1 & 1 & 1 \\
\hline 3TIME & 1 & 1 & 1 & 1 & 1 & 0 & 1 & 1 & 1 & 1 \\
\hline 4TIME & 1 & 2 & 1 & 1 & 2 & 1 & 1 & 1 & 1 & 1 \\
\hline
\end{tabular}


The result is the value at which the expected value for the ith ball (np) is the maximum, and all the xi values become $\mathrm{np}$, which is the expected value. With many iterations of random selection, each ball's frequency of selection will approach np. However, as the frequency of selection increases, the standard deviation will increase, and it will be increasingly harder to achieve uniform selection by using simple random selection [10], [11], [12].

A simple way to ensure uniform frequency of the selection of all the balls through random selection is to sort the frequency of selection in ascending order and select randomly from those balls having the lowest frequency of selection.

With this method, the frequency of selection of each ball is near the expected value with only small deviations. However, if the total number of balls is large, this method will be inefficient as it will require long computation times to sort in ascending order each time.

In summary, if you select $m$ balls at the same time, you do not want to reselect them. Although simple, these operations are not solved by a simple uniform random number generator in order to make the selection uniform as a whole. It is important to use the total number of selections at the time of the selection, and the total number of attempts should be considered every $n$ times. For this case, an algorithm has been devised that uses the frequency of selection, eliminates the process of sorting, and performs processing using only the information on the frequency of selection of the selected locations [13]. This algorithm can be understood using a topographical analysis.

\subsection{TOPOGRAPHICAL ANALYSIS FOR UNIFORM SELECTION}

If the number of times of selection for five points including two points on each side of the selected point are regarded as height values, the sequence will appear as a flat place, a downhill road, and an uphill road (Fig. 1). The purpose of the algorithm is to provide several rules to allow an automatic process for smoothing the bumpy road. If the road is smooth, the frequency of selection will be uniform, and uniform selection will be the result.

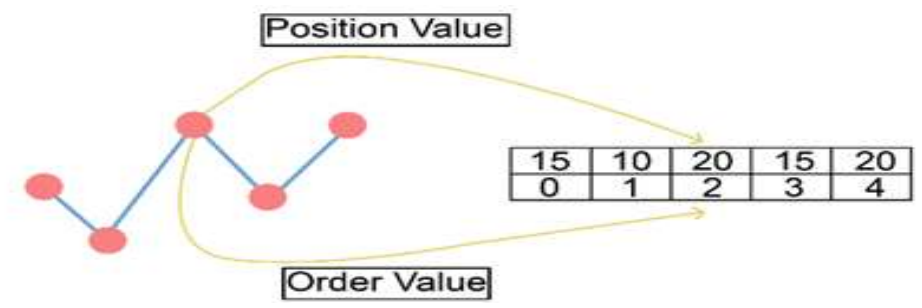

Fig. 1 Topographic Location and Value at Topographic Location [13]

The information needed by the algorithm is the following: the height of a randomly selected location and a comparison of the heights of the points immediately to the left and right of this point. From these, a determination can be made whether to move or stop. Since the two points on either side can be seen simultaneously, it is known whether the adjacent point is higher or lower. This information will be used to decide whether to move.

Figs. 2 and 3 display the topographical features used for making the decision to move from or stop at a selected location.
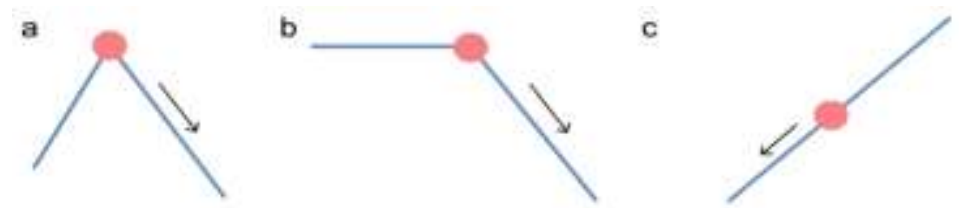

Fig. 2 (a) Movement Down the More Steeply Sloped Side (b), (c) Movement Down Sloped Side [13] 
In each configuration shown in Fig. 2, at least one of the two directions is a downward slope.
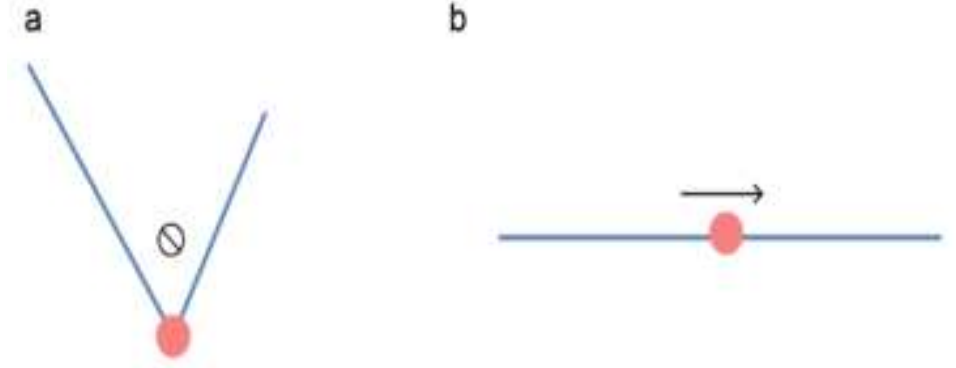

Fig. 3 (a) Case of stop (b) case of move in the forward direction. [13]

In each configuration shown in Fig. 2, at least one of the two directions are a downward slope. The decision is to move two positions in the steeper downhill direction.

In contrast, if the height in both directions is greater than that at the selected point, the height should be adjusted. If the road is flat, rather than remaining at the selected location, the algorithm moves forward and checks for a point having a lower height. If one is found, it determines the movement from that point, and if after moving five times the road is still flat, it increases the height to create a hill at the location of the previous movement. These two configurations are shown in Fig. 3 [14].

In order to handle this important task, the array that saves the number of times chosen for each point should be handled as a circular array with the ends connected. For example, if there are 10 balls, and if the selected point is the second point, then at the two points to the left are the values for the first and tenth positions, and at the two points to the right are the values for the third and the fourth positions.

In the cases described thus far, decisions about moving and stopping are made on the basis of simple rules, but there are some additional considerations for the following cases.

The configurations shown in Fig. 4 may appear to indicate a decision to stop since the selected point is lower than the point just beside it, but here the movement is decided according to the height of the points two positions away. In the case of Fig. 4(a), since the selected location is lower than those of the four points nearest to it, no further movement is necessary. However, in the case of Fig. 4(b), the point on the right side is higher, but the point at the right end is lower than the selected point. Hence, the decision is to move to the right-end point and then to stop or move according to the heights at the new surroundings.

a

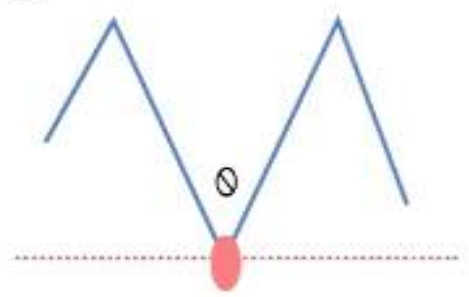

b

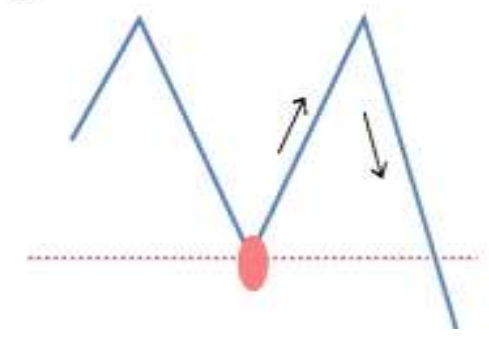

Fig. 4 Lower position (a) decision is to stop (b) decision is to move

In another configuration, shown in Fig. 5, the points in both directions from the selected point are lower, but the heights of the ending positions in both directions are greater. In this case, the decision is to move one space toward the lower point and increase the height there. 


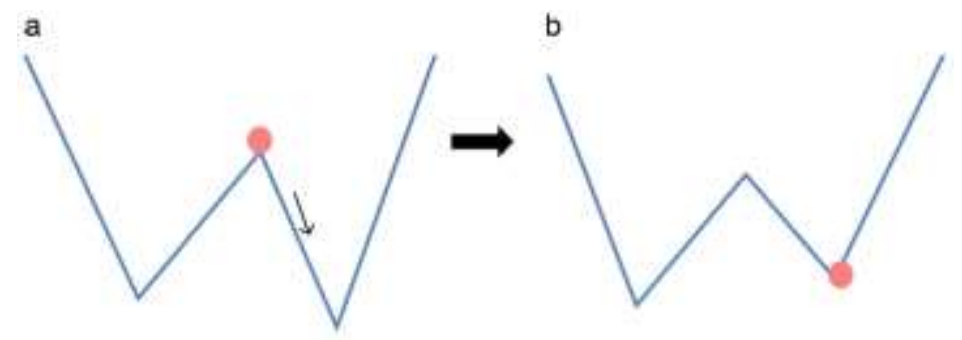

Fig. 5 (a) Decision is to Move to the Side with the Lower; (b) result is increased highposition value

For the configuration shown in Fig. 6(a), in which the two points to the right of the selected point are both lower, the decision is to move right to the first lower point and increase the height as shown in Fig. 6(b).
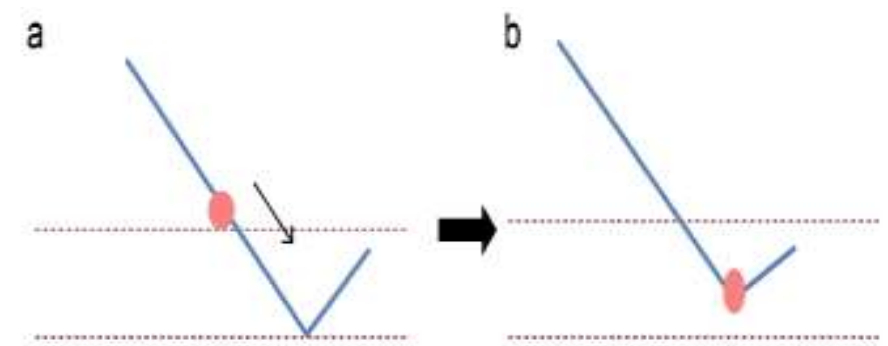

Fig. 6 (a) Only the point on one side is lower (b) result is increased high-position value

Fig. 7 shows the last configuration, in which one of the points on either side of the selected point is flat or both sides are flat. In Fig. 7(a), there is no point lower than the selected point, but if the left side is flat, the decision is to move to the flat side to determine the selection point. In Fig. 7(b), there is no point lower than the selected point and both sides are flat, but since the second point to the left is higher than the selection point and the slope is upward, the decision is to move to the right to determine the point of selection.
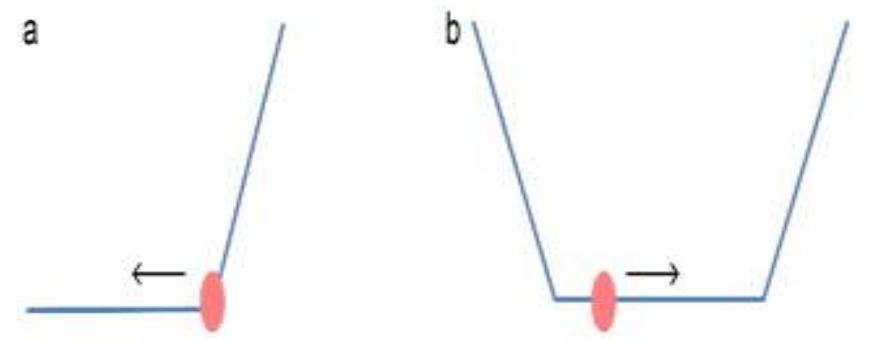

Fig. 7 The lowest point, but movement is needed (a) reverse movement (b) forward movement

One problem with this algorithm occurs in the case when all the points are high except for one point that is low. If the total number of selections is low, every time $m$ balls are chosen, this point will be continually marked as the selected point, thus falling into an infinite loop. To resolve this problem, when the same points are continually selected, an adjacent point is chosen as the selection point regardless of its height. When the specified rules are repeatedly applied, the frequency of selection will begin with a value of 0 , but through the selection process, curves and hills will be created, and the algorithm will work to automatically flatten the terrain. 
If a new addition is made in the middle and the height is 0 , the number of times selected is automatically increased. If a point is relatively high, it will not be selected until the heights of the points around it are increased; hence, no action is required for management.

As can be seen in the method for determining the direction of movement, which is to compare the height of the selected point with the heights of the two points on each side of the selected location, the processing speed for the selection process is the same regardless of the quantity of data.

\section{PSEUDOCODE FOR ALGORITHM}

samples $=$ Selection frequency value for index point.

while ( $\mathrm{i}<=$ total number of selections) \{

$\mathrm{v}=0$;

$\mathrm{idx}=(0 \sim$ total number of selections -1$)$ randomly select locations between the values.

direct $=$ The selection direction is set to be stopped.

while $(\mathrm{v}<5)\{$

obtain the height differences between the selected point and each of the two points.

if (it is in a valley) \{

if (the heights of the ending positions on each side are greater) \{

break;

\}

\} else if (the height of one of the points adjacent to the selected point is less than the other \&\& the height of the ending position in the lower direction is greater than those of the adjacent points) \{ move one space in the relevant direction.

break;

\} else if (terrain is flat) \{

move 3 spaces to the right

direct $=$ right side

$\mathrm{v}=\mathrm{v}+1$

continue;

\} else if (the adjacent points have the same height but the heights of the ending positions are greater) \{ break;

find the end of a flat region and select any spot in that region

\}

if (the previous direction of movement is different from the current direction of movement) \{ break;

\}

\}

move 2 spaces in the direction of movement

if (the selected location is the same as the previous location) \{

move one space in the steeper direction \}

if (the selected location is not in the current sample set)

\{

increase the height for the selected location

add the current selected location to the set of location samples

\}

\} [13]. 


\section{EXPERIMENTS}

\subsection{BASIC EXPERIMENT}

Table II shows the result of extracting three points from 10 points by applying this algorithm.

Table II. Example of the Application of Algorithm

\begin{tabular}{|c|c|c|c|c|c|c|c|c|c|c|}
\hline & No 1 & No 2 & No 3 & No 4 & No 5 & No 6 & No 7 & No 8 & No 9 & No 10 \\
\hline 1 time & 0 & 1 & 0 & 0 & 0 & 0 & 0 & 0 & 1 & 1 \\
\hline 2 time & 0 & 1 & 1 & 1 & 1 & 0 & 0 & 0 & 1 & 1 \\
\hline 3 time & 1 & 1 & 1 & 1 & 1 & 1 & 1 & 0 & 1 & 1 \\
\hline 4 time & 2 & 2 & 1 & 1 & 1 & 1 & 1 & 1 & 1 & 1 \\
\hline 5 time & 2 & 2 & 2 & 1 & 2 & 1 & 1 & 2 & 1 & 1 \\
\hline 6 time & 2 & 2 & 2 & 2 & 2 & 1 & 1 & 2 & 2 & 2 \\
\hline 7 time & 2 & 2 & 2 & 2 & 2 & 2 & 2 & 2 & 3 & 2 \\
\hline 8 time & 3 & 3 & 2 & 2 & 2 & 2 & 3 & 2 & 3 & 2 \\
\hline 9 time & 3 & 3 & 3 & 2 & 3 & 2 & 3 & 2 & 3 & 3 \\
\hline 10 time & 3 & 3 & 3 & 3 & 3 & 3 & 3 & 3 & 3 & 3 \\
\hline
\end{tabular}

When iterated 10 times, the expected value is 3 , and as a result, the frequency of selection appears to be 3 for all of the points. It can be seen that in the individual rows, the selection of points does not appear to have a pattern. It can also be seen that in each individual column, the selected positions are spread out in somewhat regular intervals.

For comparison, Table III shows the case of simple selection. Although the rows give the appearance of random selection, the final frequencies of selection range from 2 to 5 , and only three of the 10 points have the expected value of 3 . The distribution of the selected positions within a column can be dense or far apart. This is because the data of previous selections are not considered when making each selection.

Table III. Example Of A Simple Selection Method

\begin{tabular}{|c|c|c|c|c|c|c|c|c|c|c|}
\hline & No 1 & No 2 & No 3 & No 4 & No 5 & No 6 & No 7 & No 8 & No 9 & No 10 \\
\hline 1 time & 0 & 0 & 1 & 0 & 1 & 1 & 0 & 0 & 0 & 0 \\
\hline 2 time & 0 & 0 & 1 & 0 & 1 & 1 & 1 & 1 & 0 & 1 \\
\hline 3 time & 1 & 0 & 1 & 0 & 2 & 1 & 1 & 1 & 1 & 1 \\
\hline 4 time & 2 & 1 & 1 & 0 & 2 & 1 & 2 & 1 & 1 & 1 \\
\hline 5 time & 2 & 2 & 1 & 0 & 2 & 1 & 2 & 2 & 2 & 1 \\
\hline 6 time & 2 & 3 & 1 & 0 & 3 & 1 & 2 & 2 & 2 & 2 \\
\hline 7 time & 3 & 3 & 1 & 1 & 4 & 1 & 2 & 2 & 2 & 2 \\
\hline 8 time & 3 & 4 & 1 & 1 & 4 & 1 & 2 & 3 & 2 & 3 \\
\hline 9 time & 3 & 4 & 2 & 2 & 4 & 1 & 2 & 3 & 3 & 3 \\
\hline 10 time & 3 & 4 & 2 & 2 & 5 & 2 & 2 & 3 & 4 & 3 \\
\hline
\end{tabular}

\subsection{COMPARISON EXPERIMENTS}

Next, we performed a simulation of randomly selecting 10-20 balls at once from 1000 balls for 10000 iterations. If 10 balls were selected each time, the expected value for the number of times each ball is chosen would be 100 . If 20 were selected each time, each ball would be expected to be chosen 200 times. Thus, since 10-20 balls are selected each time, the expected value for each ball should be between 100 and 200. The experiment results are shown in Table IV. Since the selected numbers are obtained from a uniform random number generator, the average approaches 150 . Therefore, the expected value for each ball should be near 150 . 
Table IV. Choosing 10-20 Balls From 1000 Balls 10000 Times [13]

\begin{tabular}{|c|c|c|c|c|c|}
\hline & $\begin{array}{c}\text { Min no. } \\
\text { times }\end{array}$ & $\begin{array}{c}\text { Max no. } \\
\text { times }\end{array}$ & $\begin{array}{c}\text { Avg no. } \\
\text { times }\end{array}$ & $\begin{array}{c}\text { Standard } \\
\text { deviation }\end{array}$ & $\begin{array}{c}\text { Avg Selection } \\
\text { amount }\end{array}$ \\
\hline Uniform random method & 119 & 197 & 155.345 & 12.3902 & 15.5345 \\
\hline Algorithm method & 153 & 159 & 155.889 & 1.3352 & 15.5889 \\
\hline
\end{tabular}

If one judge by the average number of times each ball is selected, the difference between the two methods is not obvious as the results are almost identical; it is the number of balls selected at a time multiplied by 10 . However, the difference is apparent from the distribution of the number of times each ball is selected. Under the uniform random selection method, the range in the number of times a ball is chosen is 78 and the standard deviation is 12.3902 , but for the algorithm method, the range is $6(1 / 13$ the value of the other method) and the standard deviation is 1.3352. The histograms for the number of times a ball is chosen are given in Figs. 8 and 9. The $\mathrm{x}$-axis is the number of times a ball is chosen, and the $y$-axis is the number of balls; they form a normal distribution with a mean of about 155 .

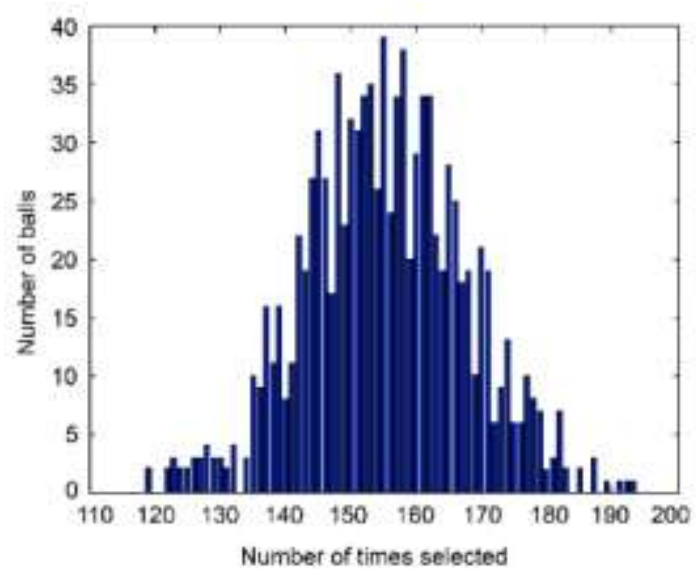

Fig. 8 Histogram for Number of Times a Ball is Chosen: Uniform Random Selection Method [13]

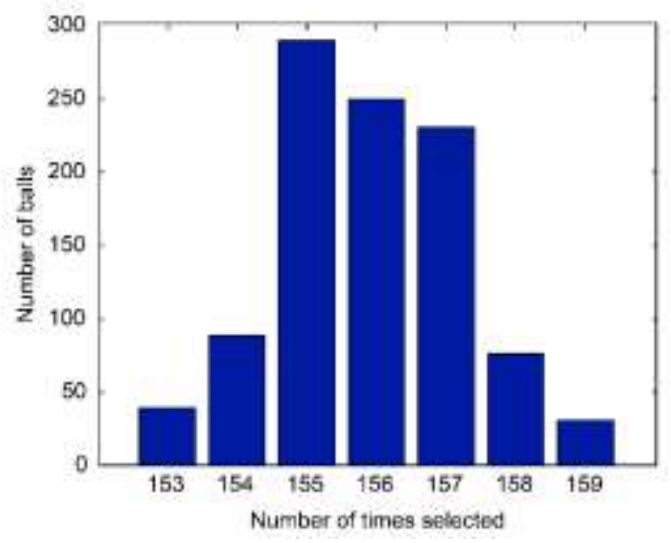

Fig. 9 Histogram for Number of Times a Ball is Chosen: Algorithm Method [13]

The next experiment explores the case when one of the frequencies of selection is large and the others are small. This is the case, for example, when three batteries are being selected each time from a set of 10 batteries, one of which is used and the rest new. A comparison was done with initial values of 0 for the frequency of selection except for the third battery, which started at 5 (Table V). 
Table V. Starting Values of Frequency of Selection for Experiment: of 10 Batteries, Only Battery 3 Starts with Five Recharges

\begin{tabular}{|l|l|l|l|l|l|l|l|l|l|}
\hline 0 & 0 & 5 & 0 & 0 & 0 & 0 & 0 & 0 & 0 \\
\hline
\end{tabular}

Under the uniform random selection method (whose results are given in Table VI), the third position is selected twice, and the final frequencies of selection range from a minimum of 1 to a maximum of 7 . In addition, the selection is not uniform. On the other hand, under the proposed algorithm (whose results are given in Table VII), when the process of selecting three batteries each time is iterated 10 times, the third battery is never selected. Hence, its final frequency of selection remains at 5. Even if there is a high value in the middle, the selection is not made until a uniform selection is made. Because it automatically focuses on the other lower selection, it is more effective in applications involving a limited number of reuses, such as a rechargeable battery.

Table VI. Experiment with One Value of Frequency of Selection Already High at The Start: Uniform Random Selection Method

\begin{tabular}{|c|c|c|}
\hline 3 & 10 & 2 \\
\hline 5 & 8 & 7 \\
\hline 3 & 6 & 7 \\
\hline 9 & 5 & 6 \\
\hline 4 & 2 & 6 \\
\hline 4 & 8 & 2 \\
\hline 1 & 8 & 6 \\
\hline 6 & 9 & 2 \\
\hline 4 & 9 & 7 \\
\hline 8 & 5 & 1 \\
\hline
\end{tabular}

\begin{tabular}{|l|l|l|l|l|l|l|l|l|l|}
\hline 2 & 4 & 7 & 3 & 3 & 5 & 3 & 4 & 3 & 1 \\
\hline
\end{tabular}

Table VII. Experiment with One Value of Frequency of Selection Already High at the Start: Algorithm Method

\begin{tabular}{|c|c|c|}
\hline 10 & 5 & 6 \\
\hline 8 & 4 & 7 \\
\hline 5 & 6 & 1 \\
\hline 9 & 7 & 2 \\
\hline 1 & 8 & 4 \\
\hline 5 & 6 & 4 \\
\hline 9 & 7 & 2 \\
\hline 10 & 5 & 9 \\
\hline 8 & 1 & 2 \\
\hline 8 & 10 & 1 \\
\hline
\end{tabular}

\begin{tabular}{|l|l|l|l|l|l|l|l|l|l|}
\hline 4 & 3 & 5 & 3 & 4 & 3 & 3 & 4 & 3 & 3 \\
\hline
\end{tabular}

Next is a comparison exploring the reverse situation: All of the batteries have already been selected three times each except the third one, which is a new battery with 0 recharges (Table VIII). 
Table VIII. Starting Values of Frequency of Selection for Experiment: of 10 Batteries, only Battery 3 Starts with 0 Recharges

\begin{tabular}{|l|l|l|l|l|l|l|l|l|l|}
\hline 3 & 3 & 0 & 3 & 3 & 3 & 3 & 3 & 3 & 3 \\
\hline
\end{tabular}

Since the uniform random selection method (whose results are given in Table IX) does not consider past data and only prevents overlapping, it does not attempt to select the third battery even though it is new. The algorithm method, however, continues selecting it until the number of usages of the third battery becomes similar to those of the others, at which point it will focus on uniform selection. After 10 iterations, each battery's frequency of selection is either 5 or 6 (Table $\mathrm{X}$ ).

Table IX. Experiment With One Value Of Frequency Of Selection Low At The Start: Uniform Random Selection Method

\begin{tabular}{|c|c|c|}
\hline 9 & 8 & 2 \\
\hline 7 & 1 & 6 \\
\hline 6 & 7 & 5 \\
\hline 7 & 4 & 3 \\
\hline 5 & 4 & 2 \\
\hline 10 & 8 & 9 \\
\hline 8 & 7 & 6 \\
\hline 9 & 5 & 1 \\
\hline 6 & 1 & 7 \\
\hline 6 & 8 & 1 \\
\hline
\end{tabular}

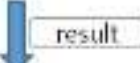

\begin{tabular}{|l|l|l|l|l|l|l|l|l|l|}
\hline 7 & 5 & 1 & 5 & 6 & 8 & 8 & 7 & 6 & 4 \\
\hline
\end{tabular}

Table X. Experiment with One Value of Frequency of Selection Low at the Start: Algorithm Method

\begin{tabular}{|c|c|c|}
\hline 3 & 4 & 6 \\
\hline 7 & 3 & 4 \\
\hline 8 & 3 & 1 \\
\hline 9 & 5 & 10 \\
\hline 2 & 3 & 8 \\
\hline 5 & 7 & 10 \\
\hline 2 & 1 & 3 \\
\hline 9 & 6 & 7 \\
\hline 6 & 10 & 8 \\
\hline 9 & 2 & 1 \\
\hline
\end{tabular}

result

\begin{tabular}{|l|l|l|l|l|l|l|l|l|l|}
\hline 6 & 6 & 5 & 5 & 5 & 6 & 6 & 6 & 6 & 6 \\
\hline
\end{tabular}

\subsection{WEIGHTS EXPERIMENT}

The method as described so far is an effective way to make uniform selections, but there is a way to make it select to fit other types of distributions: by giving weights to the values of the frequency of selection [15]. For example, focusing on the 50th ball out of 100 balls, suppose we wish to make the proportion of selections fit the shape of a normal distribution (Fig. 10). 


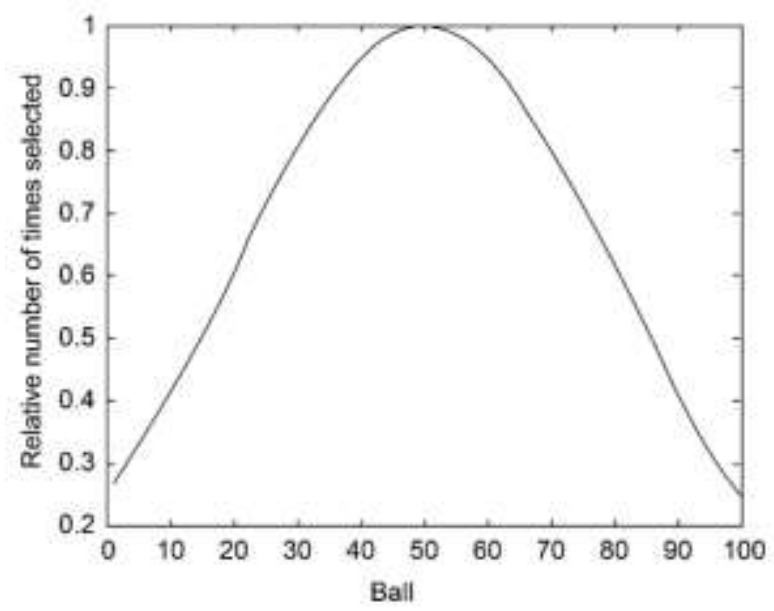

Fig. 10 Default Selection for Applying a Weight Distribution

In order to apply this weighting, the actual frequency of selection must be inversely proportional to the weight. For example, if the 50th ball has actually been selected four times, it should be made to appear as though it has been selected only once, and if it has been selected only once, it should be made to appear as though it has been selected four times. In this example, in order to change the frequency of selection to a normal distribution $N()$ with a mean of 50 and a standard deviation of 30 , the following rule should be applied:

$$
\text { actual selection count } \times \frac{N(50 \mid 50,30)}{N(\text { position } \mid 50,30)}
$$

As shown in Fig. 11, the value at the center is equal to the actual frequency of selection. The height on both sides is made to appear greater than it actually is by multiplying the actual frequency of selection by 3.8. Fig. 12 shows the result of applying this weighting and selecting three balls each time for 10000 iterations.

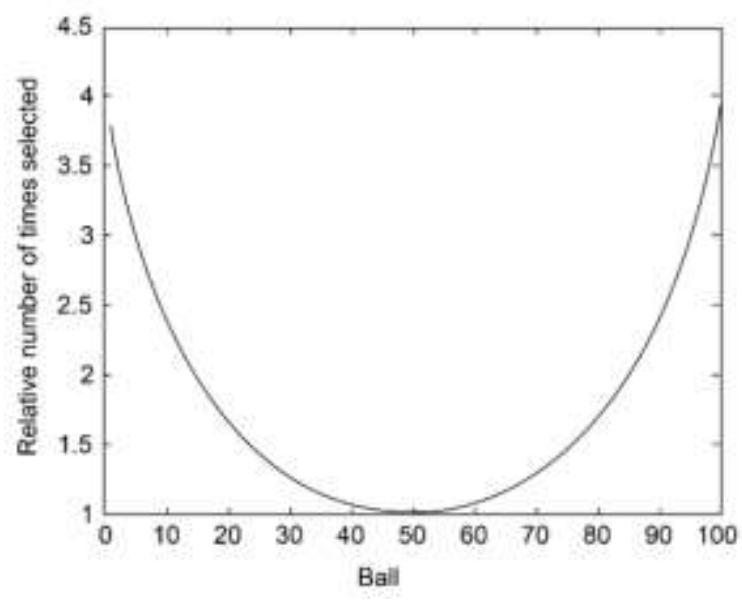

Fig. 11 Virtual Distribution should be Selected as the Default Selection 


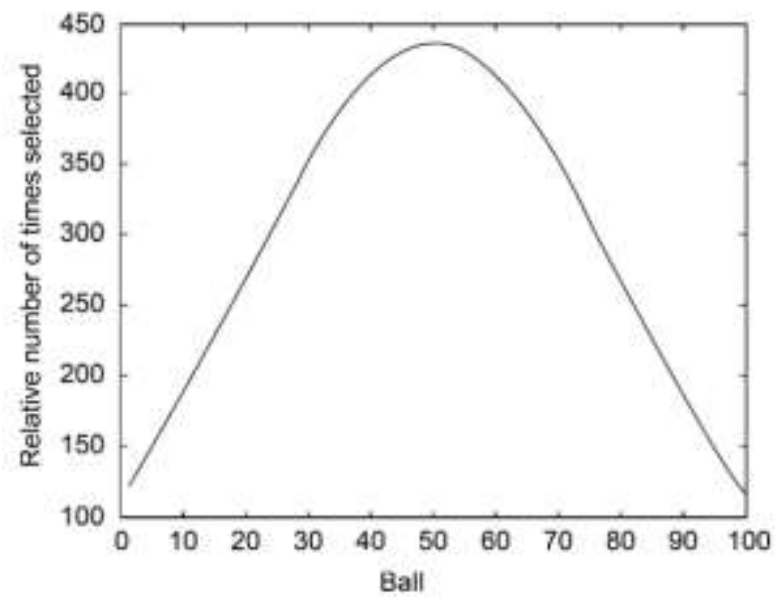

Fig. 12 Actual Distribution after Applying Weighting Algorithm

The $\mathrm{x}$-axis represents the position from 1 to 100 , and the $\mathrm{y}$-axis is the actual number of times selected. The ratio of the maximum and minimum values is 3.8 , as desired.

\section{CONCLUSIONS}

The algorithm proposed in this paper performs multi-sample selection and maintains the uniformity of the selection by applying a few simple rules using information on the number of times chosen for five points. The proposed algorithm is fast (no difference in speed), and it guarantees uniform selection whether the number of data is 100,10000 , or in the millions [16]. It can be useful in work that requires the equal treatment of big data (having more than a million points). In applications in which there is a need to add an item that has already been selected or one that has been rarely selected, the algorithm will automatically cause the total number of selections to be uniform.

The disadvantage of the simple random sampling method is that it cannot guarantee uniform selection. It is also unable to provide a selection method using different weights for the ones selected. The algorithm proposed in this paper is outstanding in terms of its efficiency in random sampling.

The algorithm is suitable for a variety of applications. A test question bank, for example, which provides randomly selected questions to a service provider, is more effective when it provides uniform exposure to all exam questions rather than focusing on just a few. In a vocabulary system, with uniform exposure, just a few words each day can be exposed from a large vocabulary set, and the number of words exposed can be adjusted by applying different weights of exposure for each word [17].

An adaptive random selector can be constructed by varying the weights according to the characteristics of an algorithm designed for uniform selection and using the result of the adjustment function as the selection frequency [18], [19]. For example, when providing test questions, at first, the level of difficulty is set to be the same for all questions, and all the questions are equally exposed. Then, the level of difficulty of each question can be adjusted using the number answered correctly by students and the weights of exposure adjusted correspondingly. In other words, if most students answer the question correctly, the weight of exposure would be decreased, and if only half get the answer right, the weight of exposure could be increased to allow it to appear more frequently. Using the data of the adjusted level of difficulty, the algorithm could also be employed to raise the confidence of low-level students by increasing the exposure weights of questions that most students answer correctly, 
providing questions in a way such that students can gradually solve higher-exposure questions.

In the case of electric cars equipped with several battery packs with limited charge cycles, battery packs can be exchanged for charged packs instead of being charged at the charging stations, and the number of usages of all the battery packs should be kept similar for purposes of the exchanging. If there are only a few battery packs at the charging station, this can be managed by humans, but for managing large numbers of battery packs or for machine automation, an algorithm becomes necessary.

This uniform selection algorithm is useful as a management technique in cases where the pool of items to be selected from is large and for systems for which maintaining the uniformity of selection is important because of physical constraints. An additional advantage is that a complicated algorithm is not necessary even when making use of the selection weight feature and even if adjustments are needed to adapt the weight for automatic selection because of changes in circumstances.

\section{ACKNOWLEDGMENTS}

This research is supported by Chung-Nam National University Research Fund.

\section{REFERENCES}

[1] Yinglei Wang, Wing-kei Yu, Shuo Wu, Greg Malysa, G. Edward Suh, Edwin C. Kan. "Flash Memory for Ubiquitous Hardware Security Functions: True Random Number Generation and Device Fingerprints." IEEE Symposium on Security and Privacy, Kota Kinabalu, Malaysia, 20-23 May 2012, pp. 3-47, IEEE Xplore, ieeexplore.ieee.org/xpl/mostRecentIssue.jsp?punumber=6233637

[2] Charles Eckert, Fatemeh Tehranipoor, John A. Chandy. "DRNG: DRAM-Based Random Number Generation Using its Startup Value Behavior." IEEE 60th International Midwest Symposium on Circuits and Systems (MWSCAS), Boston, USA, 6-9 August 2017, pp. 1260-1263, IEEE Xplore, ieeexplore.ieee.org/xpl/mostRecentIssue.jsp?punumber=8039346.

[3] Alexandros Kalousis, Julien Prados, Melanie Hilario. "Stability of Feature Selection Algorithms: A Study on High Dimensional Spaces.” Knowledge and Information Systems 12.1 (2007): 95-116.

[4] Salem Alelyani, Zheng Zhao, Huan Liu. "A Dilemma in Assessing Stability of Feature Selection Algorithms." 2011 IEEE 13th International. Conference on High Performance Computing and Communications, Banff, AB, Canada, 2-4 September 2011, pp. 701-707, IEEE Xplore, ieeexplore.ieee.org/xpl/mostRecentIssue.jsp?punumber=6062562.

[5] D. B. Thomas, Wayne Luk. "FPGA-Optimised Uniform Random Number Genera-tors Using LUTs and Shift Registers." 2010 International Conference on Field Pro-grammable Logic and Applications, Milano, Italy, 31 August - 2 September 2010, pp. 77-82, IEEE Xplore, ieeexplore.ieee.org/xpl/mostRecentIssue.jsp?punumber=5690273.

[6] D.B. Thomas, Wayne Luk, "The LUT-SR Family of Uniform Random Number Generators for FPGA Architectures." IEEE Tran. Very Large Scale Integration (VLSI) Systems 21.4 (2013): 761-770.

[7] F. Brglez, G. Gloster, G. Kedem. "Built-In Self-Test with Weighted Random Pattern Hardware." 1990 IEEE International Conference on Computer Design: VLSI in Computers and Processors, Cambridge, MA, USA, USA, 17-19 September 1990, pp. 161-166, IEEE Xplore, ieeexplore.ieee.org/xpl/mostRecentIssue.jsp?punumber=308.

[8] Sungjin Im, Shi Li. "Better Unrelated Machine Scheduling for Weighted Completion Time via Random Offsets from Non-uniform Distributions." 2016 IEEE 57th Annual Symp. on Foundations of Computer Science (FOCS), New Brunswick, NJ, USA, 9-11 October 2016, pp. 138-147, IEEE Xplore, ieeexplore.ieee.org/xpl/mostRecentIssue.jsp?punumber=7781469.

[9] Sigui Hu. "Optimum Truncated Sequential Test of Binomial Distribution." 2011 9th International Conference on Reliability, Maintainability and Safety (ICRMS), Guiyang, China, 12-15 June 2011, pp. 293-298, IEEE Xplore, ieeexplore.ieee.org/xpl/mostRecentIssue.jsp?punumber=5963915.

[10] E.W. Weisstein, "Probability." From MathWorld-A Wolfram Web Resource, 2003, http://mathworld.wolfram.com/Probability.html. Accessed 25 $5^{\text {th }}$ October 2018

[11] E.W. Weisstein, "Statistics," From MathWorld-A Wolfram Web Resource, 2006, http://mathworld.wolfram.com/Statistics.html. Accessed 25 $5^{\text {th }}$ October 2018

[12] E.W. Weisstein, "Standard Deviation," From MathWorld-A Wolfram Web Resource, 2011, http://mathworld.wolfram.com/StandardDeviation.html. Accessed $25^{\text {th }}$ October 2018

[13] Jaehoon You, Sanggook Han, Cheong Youn. "Study on the random selection algorithm based on the geographical position." Asia-Pacific Proc. of Applied Science and Engineering for Better Human Life, 16 August 2016, pp. 1-4. 
[14] Guo-Sheng Yang, Ting Wang, Huan-Long Zhang. "Eye Location Method Based on Gabor Wavelet and Topographic Feature Extraction." 2008 International Conference on Machine Learning and Cybernetics, Kunming, China, 12-15 July 2008, pp. 2857-2862, IEEE Xplore,

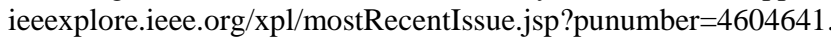

[15] Yang Xiaobo, Chen Chuxiang, Wang Zhiwan. "Improved LFM Algorithm in Weighted Network Based on Rand Walk," 2017 29th Chinese Control and Decision Conf. (CCDC), Chongqing, China, 28-30 May 2017, pp. 3719-3723, IEEE Xplore, ieeexplore.ieee.org/xpl/mostRecentIssue.jsp?punumber=7961861.

[16] Yulin He, Joshua Zhexue Huang, Hao Long, Qiang Wang, Chenghao Wei. "I-Sampling: A New BlockBased Sampling Method for Large-Scale Dataset," 2017 IEEE Internationa. Congress on Big Data (BigData Congress), Honolulu, HI, USA, 25-30 June 2017, pp. 360-367, IEEE Xplore, ieeexplore.ieee.org/xpl/mostRecentIssue.jsp?punumber=8027154.

[17] Xing-Min Chen, Chao Gao, Ming-Kun Zhang, Yi-Da Qin. "Randomized Gradient-Free Distributed Algorithms Through Sequential Gaussian Smoothing," 2017 36th Chinese Control Conference (CCC), Dalian, China, 26-28 July 2017, pp. 8407-8412, IEEE Xplore, ieeexplore.ieee.org/xpl/mostRecentIssue.jsp?punumber=8020036.

[18] Milan Vojnovi', Fei Xu, Jingren Zhou. "Sampling Based Range Partition Methods for Big Data Analytics," Technical report MSR-TR-2012-18, Microsoft Research, Redmond, WA, March 2012.

[19] T. Schoning. "A Probabilistic Algorithm for K-Sat and Constraint Satisfaction Problems," FOCS '99 Proceedings of the 40th Annual Symposium on Foundations of Computer Science, New York City, NY, USA, USA, 17-19 October 1999, pp. 410-414, IEEE Xplore, ieeexplore.ieee.org/xpl/mostRecentIssue.jsp?punumber=6604. 
International Journal of Advanced Science and Technology Vol.127 (2019) 